Bull. Mater. Sci., Vol. 6, No. 1, February 1984, pp. 27--31.

(C) Printed in India.

\title{
Magnetic structure of zinc ferrite
}

\author{
C M SRIVASTAVA, S N SHRINGI AND M VIJAYBABU \\ Department of Physics, Indian Institute of Technology, Powai, Bombay 400076 , India
}

\begin{abstract}
A detailed analysis of the available experimental data has been made for the ordered phase of $\mathrm{ZnFe}_{2} \mathrm{O}_{4}$. The abnormally low $T_{N}$ and the observed spin arrangement have been explained on the basis of the hybridization of orbitals on the oxygen ions which gives rise to an anisotropic exchange interaction. The variation of hyperfine field and susceptibility with temperature along with the zero point reduction of the magnetic moment indicate that the $\mathrm{ZnFe}_{2} \mathrm{O}_{4}$ is a two-dimensional antiferromagnet.
\end{abstract}

Keywords. Critical exponents; hyperfine fields; magnetic structure; overlap integrals; zinc ferrite.

\section{Introduction}

Zinc ferrite is a normal spinel with a cell constant of $8.44 \AA$. It is an antiferromagnetic material below $10 \mathrm{~K}$. All the $\mathrm{Zn}$ ions occupy only tetrahedral (A) site and all the $\mathrm{Fe}$ ions occupy octahedral (B) site and the only exchange interaction present in $\mathrm{ZnFe}_{2} \mathrm{O}_{4}$ is the B $B$ interaction. However, its properties like sub-lattice magnetization and susceptibility do not show a general three-dimensional (3D) antiferromagnetic behaviour. Its Néel temperature $T_{N}$ is abnormally low.

Neutron diffraction studies of $\mathrm{ZnFe}_{2} \mathrm{O}_{4}$ at $4 \cdot 2 \mathrm{~K}$ by König et al (1970) and Boucher et al (1970) have indicated that the magnetic unit cell gets doubled and becomes tetragonal with $a=b=8.43 \AA$ and $c=16.86 \AA$. The conclusions reached through these two studies are not similar. In one case (König et al 1970) there are two models which fit the intensities of lines, one collinear and the other non-collinear. In the collinear model the spins make an angle of $45^{\circ}$ with the $c$-axis. In the model of Boucher et al (1970) the collinear structure has spins pointing along [100] and [010] directions. The magnetic moments on the $\mathrm{Fe}^{3+}$ ions in both cases which are close to $4 \cdot 2 \mu_{B}$ are much smaller than the expected value of $5 \mu_{B}$ for $\mathrm{Fe}^{3+}$ ions.

Magnetic susceptibility of $\mathrm{ZnFe}_{2} \mathrm{O}_{4}$ has been measured by Lotgering (1966) and also by Arrot and Goldman (1955). At high temperatures $(T>300 \mathrm{~K})$ the reciprocal susceptibility is linear (Lotgering 1966). But below $30 \mathrm{~K}$ the inverse susceptibility is almost constant with a broad minima at $15 \mathrm{~K}$.

König et al (1970) analysed the Mössbauer spectra of $\mathrm{ZnFe}_{2} \mathrm{O}_{4}$ from 4.5 to $12 \mathrm{~K}$. These studies have given the value of $T_{N}$ as $10 \mathrm{~K}$. The value of the hyperfine field $\left(H_{\mathrm{hf}}\right)$ extrapolated to $0 \mathrm{~K}$ is $515 \mathrm{kOe}$. They have shown that the $H_{\mathrm{hf}}$ variation is markedly different from the Brillouin function $B_{5 / 2}$.

Heat capacity of $\mathrm{ZnFe}_{2} \mathrm{O}_{4}$ has been measured by Edger et al $(1957,1958)$. There is a peak in the molar heat capacity versus temperature curve around $10 \mathrm{~K}$ indicating that $T_{N}$ is $10 \mathrm{~K}$.

In figure 1, we have plotted the observed values of $T_{N}$ for several ferrites listed in table 1 as a function of the inverse of the overlap integral $\left(S^{-1}\right)$ which for qualitative 


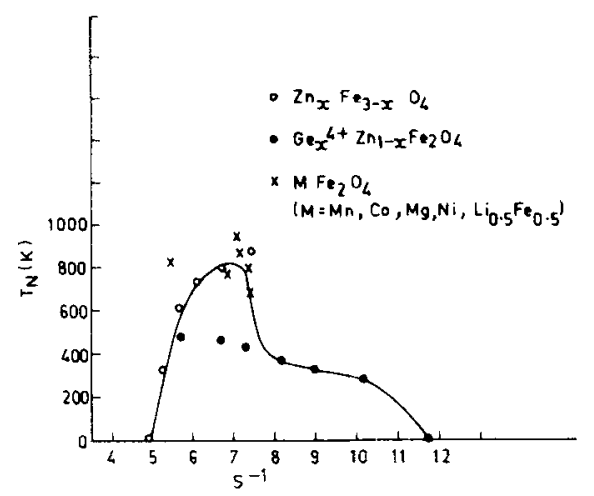

Figure 1. Variation of $T_{N}$ with the inverse of the overlap integral $S^{-1}$.

Table 1. The Néel temperature $\left(T_{N}\right)$ lattice constant $(a)$ and the inverse of the overlap integral $\left(S^{-1}\right)$ for several compositions of spinel ferrites

\begin{tabular}{|c|c|c|c|c|c|c|c|}
\hline Composition & $T_{N}(\mathbf{K})$ & $a(\AA)$ & $S^{-1}$ & Composition & $T_{N}(\mathbf{K})$ & $a(\AA)$ & $S^{-1}$ \\
\hline $\mathrm{MnFe}_{2} \mathrm{O}_{4}$ & 823 & 8.52 & $5 \cdot 44$ & $\mathrm{Zn}_{0 \cdot 6} \mathrm{Fe}_{2 \cdot 4} \mathrm{O}_{4}$ & 624 & $8 \cdot 421$ & $5 \cdot 67$ \\
\hline $\mathrm{CoFe}_{2} \mathrm{O}_{4}$ & 790 & $8 \cdot 38$ & $7 \cdot 35$ & $\mathrm{Zn}_{0 \cdot 8} \mathrm{Fe}_{2 \cdot 2} \mathrm{O}_{4}$ & 335 & 8.429 & $5 \cdot 27$ \\
\hline $\mathrm{NiFe}_{2} \mathrm{O}_{4}$ & 858 & $8 \cdot 34$ & $7 \cdot 14$ & $\mathrm{ZnFe}_{2} \mathrm{O}_{4}$ & 10 & 8.440 & 4.95 \\
\hline $\mathrm{CuFe}_{2} \mathrm{O}_{4}$ & 759 & $8 \cdot 28$ & 6.83 & $\mathrm{Ge}_{0.25} \mathrm{Zn}_{0.75} \mathrm{Fe}_{2} \mathrm{O}_{4}$ & 475 & 8.425 & 5.66 \\
\hline $\mathrm{Li}_{0.5} \mathrm{Fe}_{2.5} \mathrm{O}_{4}$ & 943 & $8 \cdot 33$ & 7.08 & $\mathrm{Ge}_{0.5} \mathrm{Zn}_{0.5} \mathrm{Fe}_{2} \mathrm{O}_{4}$ & 472 & 8.419 & 6.75 \\
\hline $\mathrm{MgFe}_{2} \mathrm{O}_{4}$ & 680 & $8 \cdot 38$ & $7 \cdot 35$ & $\mathrm{Ge}_{0 \cdot 6} \mathrm{Zn}_{0 \cdot 4} \mathrm{Fe}_{2} \mathrm{O}_{4}$ & 430 & 8.415 & $7 \cdot 32$ \\
\hline $\mathrm{Fe}_{3} \mathrm{O}_{4}$ & 875 & $8 \cdot 390$ & $7 \cdot 41$ & $\mathrm{Ge}_{0 \cdot 8} \mathrm{Zn}_{0 \cdot 2} \mathrm{Fe}_{2} \mathrm{O}_{4}$ & 325 & $8 \cdot 410$ & 8.94 \\
\hline $\mathrm{Zn}_{0 \cdot 2} \mathrm{Fe}_{2 \cdot 8} \mathrm{O}_{4}$ & 788 & $8 \cdot 399$ & 6.69 & $\mathrm{Ge}_{0 \cdot 9} \mathrm{Zn}_{0 \cdot 1} \mathrm{Fe}_{2} \mathrm{O}_{4}$ & 278 & 8.413 & $10 \cdot 18$ \\
\hline $\mathrm{Zn}_{0.4} \mathrm{Fe}_{2.6} \mathrm{O}_{4}$ & 732 & $8 \cdot 410$ & $6 \cdot 13$ & $\mathrm{GeFe}_{2} \mathrm{O}_{4}$ & 10 & 8.414 & $11 \cdot 76$ \\
\hline
\end{tabular}

estimate can be defined as

$$
S=\left[\left(r_{a}+r_{0}^{2-}\right)-\text { interatomic distance }\right] /\left[r_{a}+r_{0}^{2-}\right]
$$

where $r_{a}$ and $r_{0}^{2-}$ are the radii of the cation on the A-site and $\mathrm{O}^{2-}$ respectively and the interatomic distance is obtained from the observed cell constant $a$ and is equal to $a \sqrt{3} / 8$. Like the Slater-Bethe curve (Slater 1930; Bethe 1933) for direct overlap in metals we find that in superexchange also the large overlap leads to smaller values of exchange and as the overlap decreases the strength of exchange interaction first increases goes to a maximum and then decreases. The significant reduction in $T_{N}$ in $\mathrm{ZnFe}_{2} \mathrm{O}_{4}$ compared with $T_{N}$ of other spinel ferrites appears to be a consequence of enhanced overlap.

We have attempted to explain these experimental observations assuming that the hybridized orbitals located on the oxygen ions introduce left-right asymmetry in the 
exchange interaction. This has been explained using Anderson's model for superexchange.

\section{Discussion}

In the collinear model (König et al 1970) the alignment of spins along a few directions as shown in figure 2 is as follows:

$$
\begin{aligned}
& {[110] \text { and }[1 \overline{1} 0] \cdots \uparrow \uparrow} \\
& {[101],[\overline{101}],[011] \text { and }[01 \overline{1}]--\uparrow \downarrow \downarrow \downarrow \downarrow \uparrow \uparrow \uparrow \uparrow \downarrow \downarrow \cdots}
\end{aligned}
$$

This is a complicated structure and requires an asymmetric nearest neighbour exchange interaction. We assume that the dominant interaction between $\mathrm{Fe}^{3+}$ ions is along [110] and [1 $1 \overline{1} 0]$ directions. In this case a pair of spins ferromagnetically coupled are antiferromagnetically arranged pairwise on the linear chain. A possible explanation for the asymmetric exchange interaction on the left and right of the central spin on a linear chain has to be provided in terms of hybridized sp type of orbitals associated with $\mathrm{O}^{2-}$ ions.

The $90^{\circ} d^{5}-d^{5}$, B-B super exchange has two types of major interactions, one ferromagnetic $\left(b_{\sigma \sigma^{\prime}}\right)$ and the other anti-ferromagnetic $\left(b_{\pi \sigma}\right)$. These are shown in figure 3 .

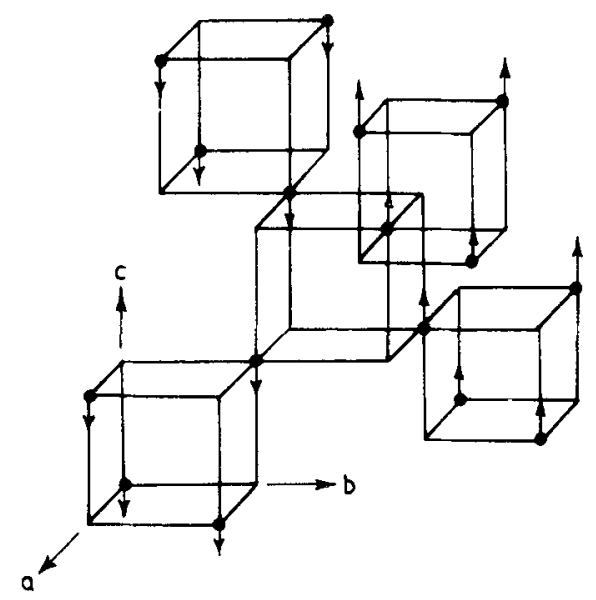

Figure 2. Spin arrangements in the collinear model (König et al 1970).

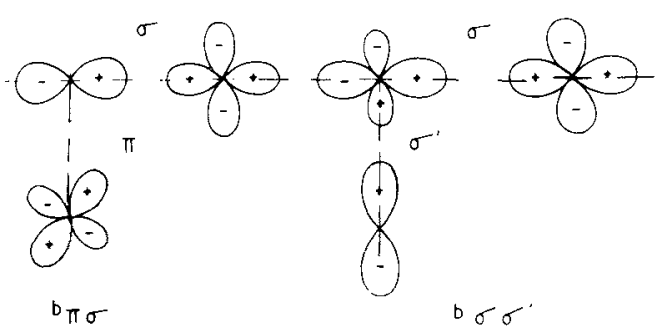

Figure 3. The $b_{\pi \sigma}$ and $b_{\sigma \sigma^{\prime}}$ transfer integrals before hybridization. 
Srivastava et al (1979) have shown that

$$
J=-(1 / 25)\left[\left(4 b_{\pi \sigma}^{2} / U\right)-\left(4 b_{\sigma \sigma^{\prime}}^{2} / U\right)\right],
$$

where $U=10 \mathrm{eV}$. If the sp hybridization occurs on the $\mathrm{O}^{2-}$ sites the strength of the $\sigma$ interaction increases while that of the $\pi$ interaction is likely to decrease as shown in figure 4. Consequently, the strength of antiferromagnetic interaction $b_{\pi \sigma}^{2} / U$ goes down while that of the ferromagnetic interaction $b_{\sigma \sigma}^{2} / U$ increases. In the case of pure orbitals without hybridization the magnitudes of $b_{\pi \sigma}$ and $b_{\sigma \sigma}$ are 0.25 and $0.122 \mathrm{eV}$ respectively (Srivastava et al 1979). The steep fall in $T_{N}$ for $\mathrm{ZnFe}_{2} \mathrm{O}_{4}$ from the case of the ferrites $\mathrm{M}_{1-x}^{2+} \mathrm{Zn}_{x} \mathrm{Fe}_{2} \mathrm{O}_{4}(x \neq 1, \mathrm{M}=\mathrm{Fe}, \mathrm{Ni}, \mathrm{Mn}, \mathrm{Co})$ shows that when $\mathrm{Zn}$ is completely occupying the A-site the effect of hybridization is most pronounced. A similar effect is observed in $\mathrm{Ge}_{x} \mathrm{Zn}_{1-x} \mathrm{Fe}_{2} \mathrm{O}_{4}$ (Miyahara and Sai 1976; Hartmann-Boutron and Imbert 1968). In this system for $x=1, \mathrm{Ge}^{4+}$ ions on the $\mathrm{A}$-site have a very small radius but they have higher ionic charge. The nearest neighbour oxygen ions are therefore attracted towards this ion. Hence, this leads to more reduction in $b_{\pi \sigma}$ compared to $b_{\sigma \sigma^{\prime}}$ and the $90^{\circ}$ $\mathrm{Fe}^{3+}-\mathrm{Fe}^{3+}$ interaction falls.

From the value of $T_{N}$ for $\mathrm{ZnFe}_{2} \mathrm{O}_{4}$ which is $10 \mathrm{~K}$ assuming the two sublattice model we obtain in the mean field approximation

$$
K T_{N}=(2 / 3)\left(-Z_{F} J_{F}+Z_{A} J_{A}\right) S(S+1),
$$

where $Z_{F}$ and $Z_{A}$ are the number of nearest neighbour spins with parallel and antiparallel arrangements and $J_{F}$ and $J_{A}$ are the strength of ferromagnetic and antiferromagnetic couplings. Taking $Z_{F}=4, Z_{A}=2$, we have

$$
J_{A}-2 J_{F}=0.86^{\mathrm{C}} \mathrm{K} \text {. }
$$

This effect of the asymmetric exchange is to reduce significantly the effective interaction and hence $T_{N}$. The spin arrangements discussed above would require that $\mathrm{ZnFe}_{2} \mathrm{O}_{4}$ should behave differently from a $3 \mathrm{D}$ antiferromagnetic system. From the observed variation of $H_{\text {hf }}$ versus $T$ data we have obtained the value of the critical exponent $\beta$ as $0 \cdot 125$. This value supports the idea that $\mathrm{ZnFe}_{2} \mathrm{O}_{4}$ is close to a $2 \mathrm{D}$ antiferromagnet (Baker 1961). The data on magnetic susceptibility (Lotgering 1966) indicating a broad maximum near the transition region and the observed magnetic moment reduction of $15 \%$ at $0 \mathrm{~K}$ (König et al 1970; Boucher et al 1970) are also in agreement (Katsumota 1977; Johnson 1981) with a lower dimensionality of the magnetic ordering of $\mathrm{ZnFe}_{2} \mathrm{O}_{4}$.

\section{Conclusion}

The present available data on $\mathrm{ZnFe}_{2} \mathrm{O}_{4}$ show that the $\mathrm{Sp}$-hybridization of oxygen ion orbitals leads to significant changes in the strength and isotropy of the $90^{\circ} \mathrm{Fe}^{3+}-\mathrm{Fe}^{3+}$

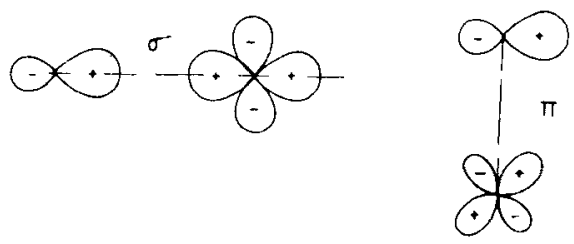

Figure 4. The $b_{\pi \sigma}$ and $b_{\sigma \sigma}$ transfer integrals after hybridization. 
superexchange interaction. The observed magnetic ordering has been attributed to these changes.

\section{Acknowledgement}

One of the authors (MV) would like to acknowledge the financial support received from CSIR; New Delhi.

\section{References}

Arrot A and Goldman J E 1955 Phys. Rev. 981201

Baker G A Jr 1961 Phys. Rev. 124768

Bethe H 1933 Handibuch der Physik 24 Pt2 Ferromagnetism 443

Boucher B, Buhl R and Perrin M 1970 Phys. Status. Soldid. 40171

Edger F, Westrum J R and Grimes D M 1957 J. Phys. Chem. Solids. 344

Edger F, Westrum J R and Grimes D M 1958 J. Phys. Chem. Sol. 6280

Hartmann-Boutron F and Imbert P 1968 J. Appl. Phys. 39775

Johnson J E 1981 Proc. Int. Conf. Mössbatuer effect, Jaipur, India

Katsumota K 1957 Solid State Commun. 23481

König U, Bertaut E F. Gros Y. Mitrikov M and Chol G 1970 Solid State Commun. 8759

Lotgering F K 1966 J. Phys. Chem. Solids 27139

Miyahara Y and Sai F 1976 J. Phys. Soc. Jpn 411522

Slater J C 1930 Phys. Rev. 3657

Srivastava C M, Srinivasan G and Nanadikar N G 1979 Phys. Ret. B19 499 